\title{
Mindfulness training for psychological stress in family caregivers of persons with dementia: a systematic review and meta-analysis of randomized controlled trials
}

\author{
This article was published in the following Dove Press journal: \\ Clinical Interventions in Aging \\ 22 September 2017 \\ Number of times this article has been viewed
}

\author{
Zheng Liu' \\ Qian-lin Chen' \\ Yu-ying Sun ${ }^{2}$ \\ 'Department of Psychiatry, The \\ Chinese University of Hong Kong, Tai \\ Po Hospital, Hong Kong SAR, China; \\ ${ }^{2}$ The Jockey Club School of Public \\ Health and Primary Care, Faculty of \\ Medicine, The Chinese University of \\ Hong Kong, Hong Kong SAR, China
}

\begin{abstract}
Caring for a relative with dementia is extremely challenging; conventional interventions may not be highly effective or easily available on some occasions. This study aimed to explore the efficacy of mindfulness training in improving stress-related outcomes in family caregivers of people with dementia using a meta-analytic review. We searched randomized controlled trials (RCT) through April 2017 from five electronic databases, and assessed the risk of bias using the Cochrane Collaboration tool. Seven RCTs were included in our review. Mindfulness interventions showed significant effects of improvement in depression (standardized mean difference: -0.58 , [95\% CI: -0.79 to -0.37$])$, perceived stress $(-0.33,[-0.57$ to -0.10$])$, and mental health-related quality of life ( 0.38 [0.14 to 0.63$])$ at 8 weeks post-treatment. Pooled evidence did not show a significant advantage of mindfulness training compared with control conditions in the alleviation of caregiver burden or anxiety. Future large-scale and rigorously designed trials are needed to confirm our findings. Clinicians may consider the mindfulness program as a promising alternative to conventional interventions.
\end{abstract}

Keywords: dementia caregivers, mindfulness, randomized controlled trials, meta-analysis

\section{Introduction}

Dementia has become a serious public health concern due to rapid population ageing. The progressively irreversible disease affects approximately 47 million people worldwide, and the number rises increasingly. ${ }^{1}$ People with dementia are affected by cognition deficits, emotional disorders, and even behavior disturbances, all of which eventually lead to inability to function independently. ${ }^{2}$ The burden of intensively caring for these dependent patients is consequently put on family caregivers in most cases, and the duration of in-home caregiving can be as long as a median of 6.5 years. ${ }^{3}$ High levels of stress are reported in virtually all dementia caregivers, predisposing this vulnerable population to develop psychological and physical morbidity, ${ }^{4}$ and even resulting in early mortality. ${ }^{5}$ The burden of caregiving can not only greatly impair the well-being and quality of life of dementia caregivers, but also be associated with a high possibility of patient institutionalization. Because of all of that, any intervention that can support caregivers to reduce their stress should be greatly welcome.

Numerous psychosocial interventions have been developed for dementia caregivers, including information provision, social support or skills training such as behavior modification techniques, etc. However, most of these achieved only mild-to-modest 
efficacy in reducing caregiver distress..$^{6-8}$ Although some treatments enjoyed stronger empirical support, such as those relying on the cognitive behavioral therapy framework (not including mindfulness-based cognitive therapy) ${ }^{9}$ and the recent ambitious interventions such as Resources for Enhancing Alzheimer's Caregivers Health, ${ }^{10,11}$ these programs are heavily dependent on humans (ie, clinical psychologists) and/or economic resources. Thus, access to these potentially effective programs can be limited because of economic, geographic, and policy barriers.

Therefore, there may not be a "one-size-fits-all" intervention that is both highly effective and easily available to various kinds of dementia caregivers. Furthermore, no matter how skillful or competent caregivers are, which is among the main goals of many conventional interventions, caregivers are bound to feel burdensome and overwhelmed from time to time. This is where the wisdom of mindfulness through strengthening inner resources likely comes in.

Borrowing wisdom from Buddhist meditation, mindfulness has aroused great interest recently in scientific literature. Mindfulness is often operationally defined as "the awareness that emerges through paying attention on purpose, in the present moment, and non-judgmentally to the unfolding of experience". ${ }^{12}$ Mindfulness can be translated in various ways in the interventions such as the mindfulness-based stress reduction, yogic meditation, and those incorporated into behavioral therapies (eg, acceptance and commitment therapies). Mounting evidence shows that mindfulnessbased programs are associated with reducing multiple negative dimensions of psychological outcomes such as perceived stress, depressive symptoms and anxiety, as well as enhancing the quality of life both acutely and chronically in a range of populations. ${ }^{13-15}$ Particularly, the individuals benefiting from mindfulness were mostly facing considerable stress in their lives; these individuals included organ transplant recipients, ${ }^{16}$ patients with cancer, ${ }^{17,18} \mathrm{HIV},{ }^{19}$ or psychological disorders. ${ }^{20,21}$ Given the potentially positive effects of mindfulness for enhancing the ability to cope with distress, it makes sense that mindfulness would also benefit dementia caregivers who are especially likely to report similar problems that mindfulness has been shown to alleviate.

Previous reviews did provide preliminary evidence of the mental benefits of mindfulness for caregivers. ${ }^{22-25}$ However, these reviews have largely included uncontrolled trials (the pre-post study without a comparison group), the results from which cannot eliminate the risk of self-selection bias. For instance, people who believe in the benefits of mindfulness are more likely to enroll in a mindfulness program and report that they benefited from one. Additionally, sample sizes of caregiver trials have been relatively small, especially in the new area of investigation such as mindfulness interventions. In this context, the value of a meta-analysis, based on aggregation of individual studies, is to increase the power of detecting the real effect as statistically significant, if it exists. Yet, most previous reviews have synthesized the results narratively without using meta-analytic techniques. . $2,24,25^{2}$ Although one previous review assessed the effectiveness of mindfulness training by using a meta-analytic technique, this study did not exclusively focus on caregivers of persons with dementia. ${ }^{23}$

In this systematic review and meta-analysis, we aimed to determine the efficacy of mindfulness training relative to control conditions in improving stress-related outcomes (depression, anxiety, perceived stress, burden, and mental health-related quality of life) in family caregivers of people with dementia.

\section{Methods}

\section{Search strategy}

We searched the Cochrane Library, MEDLINE, PsycINFO, Embase, and CINAHL. We searched these databases up until April 2017. The search terms we used pertained to mindfulness ("mindful*" or "MBCT" or "MBSR" or "mindfulnessbased cognitive therapy" or "mindfulness-based stress reduction") AND dementia ("dementia*" or "Alzheimer*”) AND caregivers ("caregiv*" or "care-giv*" or "carer*"). Search details are shown in the Supplementary materials, Tables S1-S3. In addition, we searched the reference lists of previous relevant reviews. ${ }^{22-25}$

\section{Eligibility criteria}

We included randomized controlled trials (RCTs) which compared mindfulness-based interventions with controls in family caregivers of relatives with any type of dementia living in the community setting. Studies focusing on care workers (ie, nurses) rather than family caregivers were not included. We defined mindfulness-based interventions broadly to include mindfulness-based stress reduction (MBSR) and those combining the component of mindfulness with behavioral therapies (ie, mindfulness-based cognitive therapy, acceptance and commitment therapy) or meditation-based interventions. For the comparison groups, we considered active controls, usual care or wait-list controls. In this review, the primary outcome was caregivers' levels of depression; the secondary outcomes included caregivers' levels of anxiety, perceived stress, caregiver burden, and 
mental health-related quality of life. The included studies had to measure at least one of these outcomes with psychometrically valid and reliable tools which can be used to calculate effect sizes. Additionally, included studies were written in English and published in peer-reviewed journals.

\section{Data management}

Two reviewers (ZL and QLC) independently screened the articles in two stages: at the title and abstract level and then at the full-text level. We extracted data regarding the eligibility criteria (RCT or not, targeted population, targeted outcomes); characteristics of caregivers and persons with dementia (age, gender, relationship between caregivers and persons with dementia, etc.); characteristics of interventions (sessions, duration, frequency); types of control groups and outcomes. Any disagreements were discussed between the two reviewers or solved by consulting a third reviewer (YYS) until consensus was reached.

\section{Assessment of risk of bias}

We assessed the risk of bias of individual studies using the Cochrane Collaboration risk of bias tool. ${ }^{26}$ We judged whether each study was at a low, high or unclear risk of bias for domains in selection bias, detection bias, and attrition bias. Regarding selection bias, we assessed the sequence of generation and allocation concealment. As a result of the expected small sample sizes of included trials, we also evaluated the comparability of baseline characteristics between the intervention and control groups. Concerning detection bias, we judged whether outcome assessors for our primary outcome (depression) were blinded to allocation. For attrition bias, we appraised not only the comparability of caregiver characteristics between the completers and the drop-outs, but also if the study adopted intention-to-treat analysis. In fact, it is nearly impossible to blind patients and delivery individuals in psychosocial interventions, so we did not include the assessment of performance bias or blinding of personnel and participants. Two reviewers (ZL and QLC) independently assessed the risk of bias and resolved disagreements by discussion or consultation with a third reviewer (YYS).

\section{Statistical analyses}

We entered the available data directly into RevMan version 5.3 (Cochrane Collaboration, Oxford, UK) for Mac computers. As our focused outcomes were continuous data, we calculated the standardized mean differences (SMD; effect size [ES]; Cohen's $d$ ) which were determined by mean differences of the main treatment effect between the mindfulness training group and the control group divided by the pooled standard deviation. We used immediate post-intervention values to calculate the main treatment effect. If more than one outcome measure was reported on a certain domain, we chose scales that were common to the other trials to make comparisons more meaningful. We then combined SMD using inverse variance methods and applied a random effects model for the meta-analysis as we expected a considerable amount of heterogeneity across included studies.

The statistical heterogeneity was assessed by visual inspection of forest plots, tests of significance ( $p$-value), and the $I^{2}$ statistic. The level of heterogeneity across studies was rated as low, moderate or high corresponding to $I^{2}$ value of $25 \%, 50 \%$, or $75 \%$. If moderate or high levels of heterogeneity were identified across sufficient studies which were included, we would consider conducting subgroup or meta-regression analyses based on the potential effect modifiers.

\section{Results Study selection}

A total of 248 studies were identified from searching the databases and reference lists of included studies and previous systematic reviews. ${ }^{22-25}$ After screening the titles and abstracts, eleven full-text articles were assessed for eligibility. Of these, seven studies met our inclusion criteria. A flow diagram of the selection of studies is shown in Figure 1.

\section{Description of studies}

The major characteristics of seven included studies published between 2010 to 2017 are presented in Table 1 .

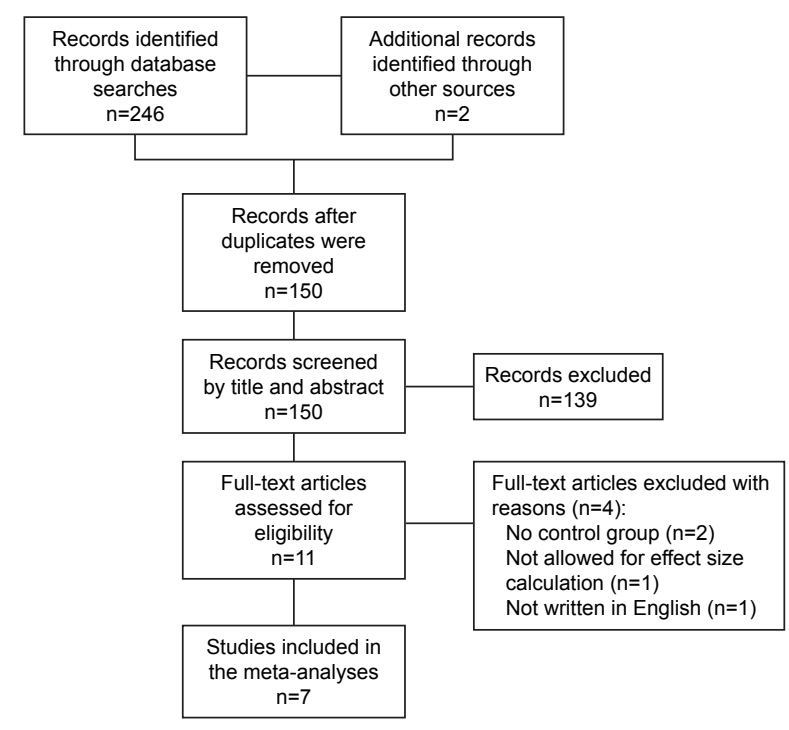

Figure I Flowchart of trial inclusion. 
Table I Characteristics of included studies

\begin{tabular}{|c|c|c|c|c|c|c|c|c|c|c|c|c|c|}
\hline \multirow[t]{2}{*}{ Study } & \multirow[t]{2}{*}{ Conditions } & \multirow[t]{2}{*}{$\mathbf{n}$} & \multirow[t]{2}{*}{ Dosage } & \multirow[t]{2}{*}{ Delivery } & \multirow[t]{2}{*}{ Age } & \multirow[t]{2}{*}{$\mathbf{W}, \%$} & \multirow[t]{2}{*}{ Sp, \% } & \multirow[t]{2}{*}{ Attr, \% } & \multicolumn{5}{|l|}{ Measures } \\
\hline & & & & & & & & & Depression & Anxiety & Stress & Burden & QoL \\
\hline \multirow[t]{2}{*}{$\begin{array}{l}\text { Brown et al, } 2016^{27} \\
\text { (USA) }\end{array}$} & MBSR & 23 & $\begin{array}{l}8^{*}(1.5-2) \mathrm{hr} / \mathrm{wk} \\
\text { a day (retreat) }\end{array}$ & G & 61.1 & 84 & 42 & $17.4 \%$ & POMS-D & - & PSS & $\mathrm{ZBI}$ & SF-36 \\
\hline & SS & 15 & $8^{*}(1.5-2) \mathrm{hr} / \mathrm{wk}$ & G & & & & & & & & & \\
\hline Hou et al, $2014^{33}$ & MBSR & 70 & $8^{*} 2 \mathrm{hr} / \mathrm{wk}$ & G & 57.5 & 83 & 40 & $8.6 \%$ & CESD & STAI & PSS & - & SF- 12 \\
\hline $\begin{array}{l}\text { (Hong Kong, } \\
\text { SAR China) }\end{array}$ & $\operatorname{lnf}$ & 71 & - & I & & & & & & & & & \\
\hline Lavretsky et al, & KK & 23 & $8 \mathrm{wk}, 12 \mathrm{~min} /$ day & I & 60.5 & 95 & 27 & $8.0 \%$ & HDRS & - & - & - & SF-36 \\
\hline $2013^{28}$ (USA) & rel & 16 & $8 \mathrm{wk}, 12 \mathrm{~min} /$ day & I & & & & & & & & & \\
\hline Losada et al, $2015^{32}$ & ACT & 33 & 8* $1.5 \mathrm{hr} / \mathrm{wk}$ & I & 61.8 & 84 & 41 & $26.7 \%$ & CESD & POMS-A & - & - & - \\
\hline (Spain) & MS & 31 & $2 \mathrm{hr}$, once & & & & & & & & & & \\
\hline Oken et al, $2010^{29}$ & MBSR & 8 & 6* $1.5 \mathrm{hr} / \mathrm{wk}$ & G & 62.5 & 81 & 74 & $20.0 \%$ & CESD & - & PSS & RMBP-C & - \\
\hline (USA) & SS & 11 & 6* $1.5 \mathrm{hr} / \mathrm{wk}$ & G & & & & & & & & & \\
\hline Waelde et al, & IR & 16 & 8* $1.5 \mathrm{hr} / \mathrm{wk}$ & G & 59.6 & 100 & - & $0.0 \%$ & CESD & - & - & - & - \\
\hline \multirow[t]{2}{*}{$2017^{30}$ (USA) } & & & $3 \mathrm{hr}$ (retreat) & & & & & & & & & & \\
\hline & TS & 15 & $6 *(10-15 \mathrm{~min}) / \mathrm{biwk}$ & 1 & & & & & & & & & \\
\hline Whitebird et al, & MBSR & 38 & $8 * 2.5 \mathrm{hr} / \mathrm{wk}$ & G & 56.8 & 89 & 26 & $2.6 \%$ & CESD & STAI & PSS & MBCB-S & SF-12 \\
\hline $2013^{31}$ & & & $5 \mathrm{hr}$ (retreat); & & & & & & & & & & \\
\hline \multirow[t]{2}{*}{ (USA) } & & & I.5 hr (“booster”) & & & & & & & & & & \\
\hline & SS & 40 & $8^{*} 2.5 \mathrm{hr} / \mathrm{wk}+5 \mathrm{hr}$ & G & & & & & & & & & \\
\hline
\end{tabular}

Notes: *Number of sessions. POMS-D, Depression subscale of the Profile of Mood States; ${ }^{34}$ POMS-A, Tension-Anxiety subscale of the Profile of Mood States; ${ }^{34}$ PSS, the Perceived Stress Scale; ${ }^{35}$ ZBI, the Zarit Burden Interview; ${ }^{36}$ SF, the mental component summary of the Health Survey; ${ }^{37-39}$ CESD, the Center for Epidemiologic Studies Depression Scale; ${ }^{40}$ STAI, the State-Trait Anxiety Inventory; ${ }^{41}$ HDRS, the Hamilton Depression Rating Scale; ${ }^{42}$ RMBP-C, the Reaction component of Revised Memory and Behavior Problems Checklist; ${ }^{43}$ MBCB-S, the subjective stress burden subscale of the Montgomery Borgatta Caregiver Burden Scale. ${ }^{44}$

Abbreviations: $n$, number of participants; W, women; Sp, spouse; Attr, attrition (the percentage of participants no long participating the mindfulness training after randomization); QoL, mental health-related quality of life; MBSR, mindfulness-based stress reduction; SS, participants received education, discussion, and social support related with dementia caregiving; Inf, participants received information related to dementia caregiving from a self-help booklet; KK, a yogic meditation named "Kirtan Kriya"; rel, relaxation, listening to music; ACT, acceptance and commitment therapy; MS, minimal support; IR, inner resources meditation including mindfulness meditation, breathfocused imaginary, and mantra repetition; TS, telephone support; hr, hour; wk, week; biwk, bi-week; min, minute; I, individual-based; G, group-based.

Five included trials were conducted in the USA; $;^{27-31}$ the others were conducted in Spain ${ }^{32}$ or Hong Kong. ${ }^{33}$ The sample size of individual studies ranged from 19 to 141 . The mean age of caregivers varied from 57 to 63 years. Women constituted the majority of caregivers (more than $80 \%$ ) in all included studies. Spousal caregivers accounted for $26 \%$ to $74 \%$ of the entire sample. All included trials reported caregivers' levels of depression, four trials assessed perceived stress and mental health-related quality of life among caregivers, and only three trials measured caregivers' level of anxiety and burden associated with caregiving.

Four trials employed a structured MBSR including six to eight weekly sessions lasting from 1.5 to 2.5 hours $;^{27,29,31,33}$ others used mindfulness programs focusing on acceptance and commitment therapy, ${ }^{32}$ yogic meditation, ${ }^{28}$ or a combination of mindfulness with mantra meditation. ${ }^{30}$ Adherence to mindfulness training was generally good; the drop-out rate for most trials was less than $20 \% .^{25-27,29-31}$ Four included studies used active controls, including education or social support, ${ }^{27,29,31}$ or listening to relaxation music. ${ }^{28}$ Other studies included control groups in which participants had decreased contact or social support from the interventionists compared with the participants in the intervention groups..$^{30,32,33}$

\section{Assessment of the risk of bias}

Table 2 summarizes the risk of bias ratings of our included studies. Although all trials were described as "randomized", the randomization technique was unclear in two studies..$^{27,30}$ All trials assessed the success of randomization by comparing the baseline variables of the comparisons, and four of them were rated as having a high risk of bias because of significant differences between the intervention and comparison groups which may have influenced intervention effects. ${ }^{27,30-32}$ For allocation concealment, we could only find clear information in two included trials. ${ }^{28,33}$ Because our primary outcome (depression) was always measured by self-rated tools, the blinding of outcome assessors was assessed as having a high risk of bias for all included trials. Among the four trials comparing the characteristics between completers and drop-outs, ${ }^{26,28,30,31}$ two of them were assessed as having a high risk of bias. ${ }^{26,28}$ More than half of the included trials conducted intention-to-treat analyses..$^{27,30,31,33}$ 
Table 2 Risk of bias ratings of the trials included in the systematic review

\begin{tabular}{|c|c|c|c|c|c|c|}
\hline Trials & $\begin{array}{l}\text { Sequence of } \\
\text { generation }\end{array}$ & $\begin{array}{l}\text { Baseline } \\
\text { comparability }\end{array}$ & $\begin{array}{l}\text { Allocation } \\
\text { concealment }\end{array}$ & $\begin{array}{l}\text { Blinding of outcome } \\
\text { assessors }\end{array}$ & $\begin{array}{l}\text { Comparability } \\
\text { of completers } \\
\text { and drop-outs }\end{array}$ & $\begin{array}{l}\text { Use of intention- } \\
\text { to-treat }\end{array}$ \\
\hline Brown et al, $2016^{27}$ & $?$ & $+^{a}$ & $?$ & + & $?$ & - \\
\hline Hou et al, $2014^{33}$ & - & - & - & + & $+^{\mathrm{b}}$ & - \\
\hline Lavretsky et al, $2013^{28}$ & - & - & $?$ & + & $?$ & + \\
\hline Losada et al, $2015^{32}$ & - & $+^{c}$ & $?$ & + & $+^{d}$ & + \\
\hline Oken et al, $2010^{29}$ & - & - & - & + & $?$ & + \\
\hline Waelde et al, $2017^{30}$ & $?$ & $t^{e}$ & $?$ & + & - & - \\
\hline Whitebird et al, $2013^{31}$ & - & $t^{f}$ & $?$ & + & - & - \\
\hline
\end{tabular}

Notes: +: High risk of bias; -: low risk of bias; ?: unclear risk of bias. ${ }^{\mathrm{a}}$ The percentage of primary caregivers in the intervention group was significantly higher than that in the control group $(p<0.05)$. ${ }^{\text {TT }}$ The drop-outs $(n=27)$ were significantly younger $(p<0.05)$ and had lower levels of physical activity than the participants $(n=1$ I 4$)$. ${ }^{c}$ The mean time of caring (years) was longer in the control group than in the intervention group $(p<0.05)$. ${ }^{\mathrm{d}}$ Completers spent fewer hours caring and had lower levels of depression at baseline than the drop-outs $(p<0.05)$. ${ }^{\text {TT }}$ The baseline log cortisol slope was flatter in the intervention group than the control group, indicating a higher stress level of participants in the intervention group. ${ }^{\mathfrak{f}}$ The baseline level of anxiety was significantly lower in the intervention group than in the control group.

\section{Effects of mindfulness-based interventions}

As presented in Table 3 and Figure 2, we reported the combined effects of mindfulness-based interventions compared to control conditions for dementia caregivers.

\section{Effects of mindfulness training on depression of dementia caregivers}

The pooled results showed that mindfulness training significantly decreased caregivers' levels of depression (seven studies; 410 participants; SMD: $-0.58,95 \%$ CI: -0.79 to -0.37 ) compared with the control groups, with small heterogeneity across studies $\left(I^{2}=7 \%\right){ }^{27-33}$

\section{Effects of mindfulness training on anxiety of dementia caregivers}

The pooled effects of mindfulness training compared with controls in reducing caregivers' levels of anxiety were not significant (three studies; 283 participants; SMD: -0.35 , 95\% CI: -0.71 to 0.01$)$, with moderate heterogeneity across studies $\left(I^{2}=54 \%\right) .{ }^{31-33}$

\section{Effects of mindfulness training on perceived stress of dementia caregivers}

The combined results showed that compared with control conditions, mindfulness training significantly reduced

Table 3 Effect of mindfulness training for dementia caregivers

\begin{tabular}{lllllll}
\hline Outcomes & $\mathbf{N}^{\mathbf{a}}$ & $\mathbf{N}^{\mathbf{b}}$ & $\mathbf{E S}^{\mathbf{c}}$ & $\mathbf{9 5 \%} \mathbf{C l}$ & $\mathbf{p}$-value & $\mathbf{I}^{\mathbf{2}}$ \\
\hline Depression & 7 & 410 & -0.58 & $-0.79,-0.37$ & $<0.00 \mathrm{I}$ & $\mathbf{7 \%}$ \\
Anxiety & 3 & 283 & -0.35 & $-0.7 \mathrm{I}, 0.0 \mathrm{I}$ & 0.060 & $54 \%$ \\
Perceived stress & 4 & 276 & -0.33 & $-0.57,-0.10$ & 0.006 & $0 \%$ \\
Caregiver burden & 3 & 134 & -0.08 & $-0.42,0.26$ & 0.530 & $0 \%$ \\
QoL* & 4 & 296 & 0.38 & $0.14,0.63$ & 0.002 & $9 \%$ \\
\hline
\end{tabular}

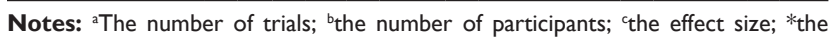
mental health-related quality of life (QoL). perceived stress, with little heterogeneity across studies (four studies; 276 participants; SMD: $-0.33,95 \% \mathrm{CI}:-0.57$ to $\left.-0.10 ; I^{2}=0\right) .{ }^{27,29,31,33}$

\section{Effects of mindfulness training on caregiver burden} The advantage of mindfulness training was not significant in comparison with the controls, with little heterogeneity across studies (three studies; 134 participants; SMD: -0.08 , $95 \%$ CI: -0.42 to $\left.0.26 ; I^{2}=0 \%\right)^{27,29,31}$

Effects of mindfulness training on mental healthrelated quality of life of dementia caregivers

The effects of mindfulness training on enhancing the mental health-related quality of life were significant relative to controls, with small heterogeneity across studies (four studies; 296 participants; SMD: 0.38, 95\% CI: 0.14 to 0.63 ; $\left.I^{2}=9 \%\right){ }^{27,28,31,33}$

\section{Discussion}

Family caregivers of people with dementia are often under considerable stress, and conventional interventions may not always be effective or feasible in reducing distress of this vulnerable population. The present review was among the first meta-analyses to quantitatively assess whether mindfulness training, novel for its brevity and cost-effectiveness, could specifically benefit caregivers of individuals with dementia. Our review indicates that mindfulness training could potentially provide stress relief, depression management, and enhance quality of life of caregivers. No significant effect was found for alleviation of caregivers' anxiety or burden associated with caregiving.

Mindfulness-based interventions have shown considerable success in improving mental health in a range of 


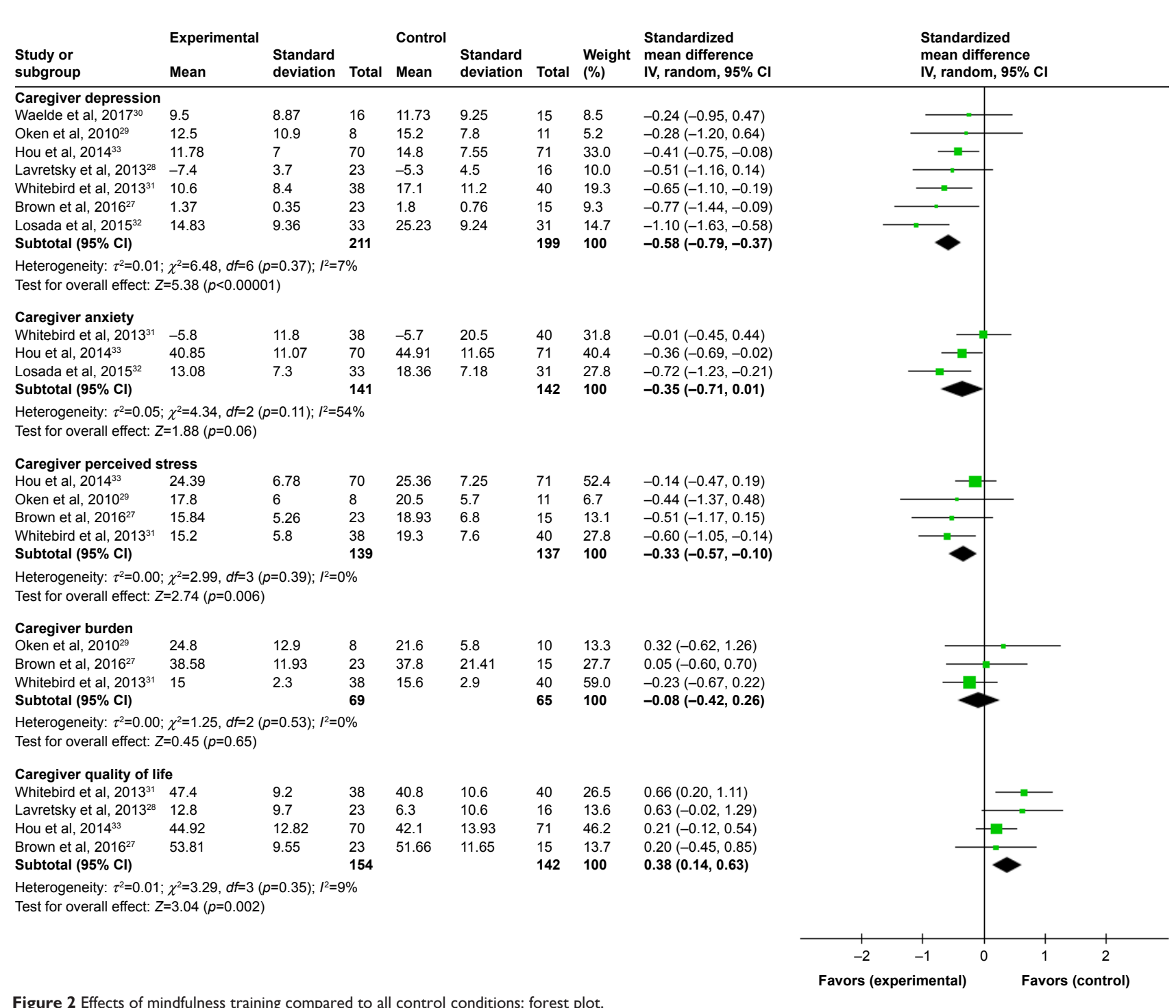

Figure 2 Effects of mindfulness training compared to all control conditions: forest plot.

populations. ${ }^{13-15}$ The consistent and relatively strong level of efficacy across varied types of samples indicates that mindfulness training might enhance coping ability in everyday life and even under more extraordinary conditions of serious stress, such as caregiving. The results from the present meta-analysis, which focuses exclusively on caregivers of persons with dementia, are generally consistent with other reviews studying caregivers of individuals with advanced illness (included but not limited to dementia). ${ }^{22-24}$

The way in which mindfulness training affects several dimensions of psychological stress among caregivers is likely under debate but may originate from the following assumptions. First, caregivers of persons with dementia, under chronic stress for several years, could have difficulties changing their automatic and habitual ways of responding to round-the-clock caregiving. ${ }^{45}$ Mindfulness-based treatments provide an opportunity for caregivers to be aware of their unhelpful reactions or thought patterns and then detach from this "automatic pilot". ${ }^{45}$ Second, there are few tangible solutions to many real-life scenarios when caring for elders with currently incurable diseases. ${ }^{45}$ Non-judgmental acceptance, ${ }^{46}$ an important element of mindfulness, can help participants "embrace" stressful caregiving as it is, but still enjoy a full and rich life.

More attention should be paid to effectiveness of mindfulness interventions on caregivers' anxiety. Our pooled result showed a non-significant trend of benefit from mindfulness training (SMD: $-0.35,95 \% \mathrm{CI}:-0.71$ to 0.01 ; $p=0.06$ ), which was inconsistent with a significant finding $(p<0.05)$ from another meta-analysis. ${ }^{23}$ Another issue is that the available evidence studying anxiety has a considerable level of statistical heterogeneity (eg, $I^{2}>50 \%$ or $Q=12.8$ ). ${ }^{23}$ The inconsistency, heterogeneity, as well as insufficiency of results in studying caregivers' anxiety, potentially limit the 
strength of available evidence regarding beneficial effects of mindfulness programs on caregivers' anxiety.

With regard to caregiver burden, we did not find a significant advantage of mindfulness training over controls in reducing burden associated with caregiving. One possibility is that caregiver burden is such a multicomponent measure (eg, the Zarit Burden Interview), ${ }^{36}$ which includes both objective and subjective aspects of caregiving, that a brief intervention like mindfulness training can hardly target the multiple features represented by the concept of burden. Keeping in mind that included trials were insufficient and heterogeneous (eg, measures on burden), our result regarding caregiver burden should be treated very cautiously.

It is important to note that the promising mindfulness training seems well-accepted by dementia caregivers. At least $70 \%$ of participants completed all of the sessions in the mindfulness-based trials, ${ }^{28,30,31}$ demonstrating reasonable adherence to the intervention. One included study arranged an orientation meeting to build enthusiasm at the beginning in addition to ongoing telephone calls and in-person contact throughout the intervention. ${ }^{31,47}$ Participants in another study learned to develop "an action plan" in the first class which encouraged them to practice activities following the intervention..$^{29}$ Of particular importance for future studies is to overcome the barriers of recruiting and retaining caregivers in novel interventions such as mindfulness training. Multipronged strategies may be needed, including the promotion of the benefit of this complementary therapy and full consideration of the role of busy family caregivers and their significant time commitment.

Finally, our study results should be weighted cautiously considering the relatively small number of trials that were included. Owing to variations in mindfulness training, our pooled results could have been influenced by heterogeneity across trials such as different content or forms of interventions. Moreover, the limited number of included trials make the power of meta-regression or subgroup analyses too low to explore potential effect modifiers which may contribute to heterogeneity across trials. Risk of publication bias can hardly be statistically assessed by tests for funnel plot asymmetry. As Cochrane Handbook suggested, the power of the tests is too low when less than ten studies are included in the meta-analysis. ${ }^{48}$ Additionally, due to the limited number of investigations with follow-up data, the meta-analysis was restricted to the immediate effects post-intervention.

Despite these limitations, our results, based on the best available evidence (RCTs), show that mindfulness training is both potentially effective and feasible for reducing several domains of psychological stress in distressed caregivers of individuals with dementia. Given the promising results of our study, future large-scale and rigorously designed trials are needed to confirm our findings, especially for the specific effects of mindfulness in reducing caregiver burden or anxiety. Clinicians may consider mindfulness training as a brief and cost-effective alternative or adjunctive treatment to conventional interventions for dementia caregivers.

\section{Acknowledgments}

We would like to express sincere gratitude to Prof Linda Chiu-Wa Lam, Prof Sing Lee, and Prof Arthur Dun-Ping Mak for their patient guidance for this manuscript preparation.

\section{Disclosure}

The authors report no conflicts of interest in this work.

\section{References}

1. Prince M, Wimo A, Guerchet M, Ali G-C, Wu Y-T, Prina M. World Alzheimer Report 2015: The Global Impact of Dementia. An Analysis of Prevalence, Incidence, Costs and Trends. London: Alzheimer's Disease International; 2015. Available from: http://www.alz.co.uk/ research/statistics.htnril. Accessed August 24, 2017.

2. Alzheimer's Association. 2016 Alzheimer's Disease Facts and Figures. Vol 12. Alzheimer's Association; 2016.

3. Haley WE. The family caregiver's role in Alzheimer's disease. Neurology. 1997;48(5 Suppl 6):S25-S29.

4. Schulz R, O’Brien AT, Bookwala J, Fleissner K. Psychiatric and physical morbidity effects of dementia caregiving: prevalence, correlates, and causes. Gerontologist. 1995;35(6):771-791.

5. Schulz R, Beach SR. Caregiving as a risk factor for mortality: the Caregiver Health Effects Study. JAMA. 1999;282(23):2215-2219.

6. Jensen M, Agbata IN, Canavan M, McCarthy G. Effectiveness of educational interventions for informal caregivers of individuals with dementia residing in the community: Systematic review and metaanalysis of randomised controlled trials. Int J Geriatr Psychiatry. 2015; 30(2):130-143.

7. Pinquart M, Sörensen S. Helping caregivers of persons with dementia: which interventions work and how large are their effects? Int Psychogeriatr. 2006;18(4):577-595.

8. Weinbrecht A, Rieckmann N, Renneberg B. Acceptance and efficacy of interventions for family caregivers of elderly persons with a mental disorder: a meta-analysis. Int Psychogeriatr. 2016;28(10):1615-1629.

9. Gallagher-Thompson D, Coon DW. Evidence-based psychological treatments for distress in family caregivers of older adults. Psychol Aging. 2007;22(1):37-51.

10. Belle SH, Burgio L, Burns R, et al. Enhancing the quality of life of dementia caregivers from different ethnic or racial groups: a randomized, controlled trial. Ann Intern Med. 2006;145(10):727-738.

11. Elliott AF, Burgio LD, Decoster J. Enhancing caregiver health: findings from the resources for enhancing Alzheimer's caregiver health II intervention. J Am Geriatr Soc. 2010;58(1):30-37.

12. Kabat-zinn J. Mindfulness-based interventions in context: past, present, and future. Clinical Psychology Science and Pracicet. 2003;10(2): 144-156.

13. Goyal M, Singh S, Sibinga EM, et al. Meditation programs for psychological stress and well-being. JAMA Intern Med. 2014;174(3): 357-368.

14. Grossman P, Niemann L, Schmidt S, Walach H. Mindfulness-based stress reduction and health benefits. A meta-analysis. J Psychosom Res. 2004;57(1):35-43. 
15. Khoury B, Lecomte T, Fortin G, et al. Mindfulness-based therapy: a comprehensive meta-analysis. Clin Psychol Rev. 2013;33(6): $763-771$.

16. Gross CR, Kreitzer MJ, Thomas W, et al. Mindfulness-based stress reduction for solid organ transplant recipients: a randomized controlled trial. Altern Ther Heal Med. 2010;16(5):30-38.

17. Henderson VP, Clemow L, Massion AO, Hurley TG, Druker S, Hebert JR. The effects of mindfulness-based stress reduction on psychosocial outcomes and quality of life in early-stage breast cancer patients: a randomized trial. Breast Cancer Res Treat. 2012;131(1):99-109.

18. Nakamura Y, Lipschitz DL, Kuhn R, Kinney AY, Donaldson GW. Investigating efficacy of two brief mind-body intervention programs for managing sleep disturbance in cancer survivors: a pilot randomized controlled trial. J Cancer Surviv. 2013;7(2):165-182.

19. Chhatre S, Metzger DS, Frank I, et al. Effects of behavioral stress reduction transcendental meditation intervention in persons with HIV. AIDS Care. 2013;25(10):1291-1297.

20. Lee SH, Ahn SC, Lee YJ, Choi TK, Yook KH, Suh SY. Effectiveness of a meditation-based stress management program as an adjunct to pharmacotherapy in patientswith anxiety disorder. J Psychosom Res. 2007; 62(2):189-195.

21. Chiesa A, Mandelli L, Serretti A. Mindfulness-based cognitive therapy versus psycho-education for patients with major depression who did not achieve remission following antidepressant treatment: a preliminary analysis. J Altern Complement Med. 2012;18(8):756-760.

22. Hurley RV, Patterson TG, Cooley SJ. Meditation-based interventions for family caregivers of people with dementia: a review of the empirical literature. Aging Ment Heathl. 2014;18(3):281-288.

23. Dharmawardene M, Givens J, Wachholtz A, Makowski S, Tjia J. A systematic review and meta-analysis of meditative interventions for informal caregivers and health professionals. BMJ Support Palliat Care. 2016;6(2):160-169.

24. Jaffray L, Bridgman H, Stephens M, Skinner T. Evaluating the effects of mindfulness-based interventions for informal palliative caregivers: A systematic literature review. Palliat Med. 2016;30(2):117-131.

25. Li G, Yuan H, Zhang W. The effects of mindfulness-based stress reduction for family caregivers: systematic review. Arch Psychiatr Nurs. 2016; 30(2):292-299.

26. Higgins JP, Altman DG, Gotzsche PC, et al. The Cochrane Collaboration's tool for assessing risk of bias in randomised trials. BMJ. 2011;343:d5928.

27. Brown KW, Coogle CL, Wegelin J. A pilot randomized controlled trial of mindfulness-based stress reduction for caregivers of family members with dementia. Aging Ment Health. 2016;20(11):1157-1166.

28. Lavretsky H, Epel ES, Siddarth P, et al. A pilot study of yogic meditation for family dementia caregivers with depressive symptoms: Effects on mental health, cognition, and telomerase activity. Int J Geriatr Psychiatry. 2013;28(1):57-65.

29. Oken BS, Fonareva I, Haas M, et al. Pilot controlled trial of mindfulness meditation and education for dementia caregivers. J Altern Complement Med. 2010;16(10):1031-1038.

30. Waelde LC, Meyer H, Thompson JM, Thompson L, GallagherThompson D. Randomized controlled trial of Inner Resources meditation for family dementia caregivers. J Clin Psychol. Epub 2017 Mar 6.
31. Whitebird RR, Kreitzer M, Crain AL, Lewis BA, Hanson LR, Enstad CJ. Mindfulness-based stress reduction for family caregivers: a randomized controlled trial. Gerontologist. 2013;53(4):676-686.

32. Losada A, Marquez-Gonzalez M, Romero-Moreno R, et al. Cognitivebehavioral therapy (CBT) versus acceptance and commitment therapy (ACT) for dementia family caregivers with significant depressive symptoms: results of a randomized clinical trial. J Consult Clin Psychol. 2015;83(4):760-772.

33. Hou RJ, Wong SY, Yip BH, et al. The effects of mindfulness-based stress reduction program on the mental health of family caregivers: a randomized controlled trial. Psychother Psychosom. 2014;83(1):45-53.

34. McNair DM, Lorr M, Droppleman LF. Profile of Mood States. San Diego, CA: Educational and Industrial Testing Service; 1981.

35. Cohen S, Karmarck T, Mermelstein R. A global measure of perceived stress. J Heal Soc Behav. 1983;24(4):385-396.

36. Zarit SH, Reever KE, Bach-Peterson J. Relatives of the impaired elderly: correlates of feelings of burden. Gerontologist. 1980;20(6): 649-655.

37. McHorney CA, Ware JE Jr, Lu JF, Sherbourne CD. The MOS 36-item Short-Form Health Survey (SF-36): III. Tests of data quality, scaling assumptions, and reliability across diverse patient groups. Med Care. 1994;32(1):40-66.

38. Ware JE Jr, Sherbourne CD. The MOS 36-item short-form health survey (SF-36). I. Conceptual framework and item selection. Med Care. 1992;30(6):473-483.

39. Lam CL, Tse EY, Gandek B. Is the standard SF-12 health survey valid and equivalent for a Chinese population? Qual Life Res. 2005;14(2): 539-547.

40. Radloff LS. The CES-D scale: a self-report depression scale for research in the general population. Appl Psychol Meas. 1977;1(3): $385-401$.

41. Spielberger CD, Gorusch RL, Lushehe R, Vagg PR, Jacobs GA. Manual for the State-Trait Anxiety Inventory. Mountain View, CA: Consulting Psychologist Press; 1983.

42. Hamilton M. A rating scale for depression. J Neurol Neurosurg Psychiatry. 1960;23:56-62.

43. Teri L, Truax P, Logsdon R, Uomoto J, Zarit S, Vitaliano PP. Assessment of behavioral problems in dementia: The Revised Memory and Behavior Problems Checklist. Psychol Aging. 1992;7(4):622-631.

44. Montgomery RJ, Borgatta EF. Societal and Family Change in the Burden of Care. Singapore: The National University of Singapore Press; 2000.

45. Mackenzie CS, Poulin PA. Living with the dying: using the wisdom of mindfulness to support caregivers of older adults with dementia. Int $J$ Health Promot Educ. 2006;44(1):43-47.

46. Lindsay EK, Creswell JD. Mechanisms of mindfulness training: Monitor and Acceptance Theory (MAT). Clin Psychol Rev. 2017;51:48-59.

47. Whitebird RR, Kreitzer MJ, Lewis BA, et al. Recruiting and retaining family caregivers to a randomized controlled trial on mindfulness-based stress reduction. Contemp Clin Trials. 2011;32(5):654-661.

48. Sterne JA, Egger M, Moher D, Boutron I, editors. Addressing reporting biases. In: Higgins JP, Churchill R, Chandler J, Cumpston MS, editors. Cochrane Handbook for Systematic Reviews of Interventions Version 5.2.0. Cochrane; 2017. 


\section{Supplementary materials Search strategy in this systematic review}

Table SI Search strategy in the Cochrane Library

I MeSH descriptor: [Mindfulness] explode all trees

2 mindful*:ti, ab, kw (Word variations have been searched)

3 MBSR:ti,ab,kw (Word variations have been searched)

4 MBCT:ti,ab,kw (Word variations have been searched)

5 I or 2 or 3 or 4

$6 \mathrm{MeSH}$ descriptor: [Caregivers] explode all trees

7 carer*:ti,ab,kw (Word variations have been searched)

8 caregiv*:ti,ab, kw (Word variations have been searched)

9 care-giv*:ti,ab,kw (Word variations have been searched)

106 or 7 or 8 or 9

II MeSH descriptor: [Dementia] explode all trees

$12 \mathrm{MeSH}$ descriptor: [Alzheimer Disease] explode all trees

13 dement*:ti,ab,kw (Word variations have been searched)

14 alzheimer*:ti,ab,kw (Word variations have been searched)

1511 or 12 or 13 or 14

165 and 10 and 15

Abbreviations: MBSR, mindfulness-based stress reduction; MBCT, mindfulnessbased cognitive therapy.

Table S2 Search strategy in Medline, Medline In-Process, Embase, and PsycINFO

\footnotetext{
I exp Mindfulness/

2 MBSR.mp.

3 MBCT.mp.

4 mindful*.mp.

5 I or 2 or 3 or 4

6 Exp Caregivers/

7 caregiv*.mp.

8 carer.mp.

9 “care-giv*”.ab,ot,ti

106 or 7 or 8 or 9

II exp Dementia/

$12 \exp$ Alzheimer Disease/

13 dement*.mp.

14 alzheimer*.mp.

1511 or 12 or 13 or 14

165 and 10 and 15
}

Abbreviations: MBSR, mindfulness-based stress reduction; MBCT, mindfulnessbased cognitive therapy.
Table S3 Search strategy in the CINAHL Complete and Plus

\begin{tabular}{ll}
\hline SI & (MH "Dementia+") \\
S2 & (MM “Alzheimer's Disease") \\
S3 & AB dement* \\
S4 & AB alzheimer* \\
S5 & SI OR S2 OR S3 OR S4 \\
S6 & (MM "Caregivers") \\
S7 & AB caregiv* \\
S8 & AB care-giv* \\
S9 & AB carer* \\
SI0 S6 OR S7 OR S8 OR S9 \\
SII (MM "Mindfulness") \\
SI2 AB mindful* \\
SI3 AB MBSR \\
SI4 AB MBCT \\
SI5 SII OR SI2 OR SI3 OR SI4 \\
SI6 S5 AND SI0 AND SI5
\end{tabular}

Abbreviations: MBSR, mindfulness-based stress reduction; MBCT, mindfulnessbased cognitive therapy.
Clinical Interventions in Aging

\section{Publish your work in this journal}

Clinical Interventions in Aging is an international, peer-reviewed journal focusing on evidence-based reports on the value or lack thereof of treatments intended to prevent or delay the onset of maladaptive correlates of aging in human beings. This journal is indexed on PubMed Central, MedLine,

\section{Dovepress}

CAS, Scopus and the Elsevier Bibliographic databases. The manuscript management system is completely online and includes a very quick and fair peer-review system, which is all easy to use. Visit http://www.dovepress. com/testimonials.php to read real quotes from published authors. 